\title{
Estrategias de ordenamiento territorial sostenible para el cantón Gualaceo
}

\section{Sustainable territorial management strategies for the Gualaceo canton}

Sandra Marisol Sarango Simbaña. ${ }^{1}$, Marco Benigno Ávila Calle. ${ }^{2}$ \& Yonimiler Castillo Ortega. $^{3}$

\begin{abstract}
.
DOI: https://doi.org/10.33262/concienciadigital.v4i2.1627

In Ecuador, since 2010, planning experiences have been given through development plans and territorial organization in charge of the Decentralized Autonomous Governments in the case of the Gualaceo canton located in the eastern part of the Azuay province. Aim. strengthen and implement strategies for land use planning that guarantees its sustainability. Methodology. Once the theoretical framework of planning and land use has been reviewed, it is necessary to reflect on its compliance with the evaluation of the PDOT in a given period 2015-2019, based on a documentary review, surveys and interviews of the perception of its authorities and planning technicians. Results. From the analysis of the research, showing a low degree of compliance in each of the strategies proposed in the phase of the proposal regarding the territorial model and the management model, which allows establishing a proposal for the environmental strategy and the strengthening of the strategies outlined in their plans. Conclusion. To guarantee a sustainable land use planning, understanding the vision of the canton with governmental leadership aware of the knowledge of the potentials and weaknesses of its territory.
\end{abstract}

Keywords: planining, local development, land use planning, management strategies.

\footnotetext{
${ }^{1}$ Universidad de Cuenca, Facultad de Arquitectura, Universidad Católica de Cuenca, Posgrado Maestría en Desarrollo Local Mención Planificación, Desarrollo y Ordenamiento Territorial, Cuenca Ecuador, sandrysarango76@ hotmail.com, https://orcid.org/0000-0001-8836-9176

2 Universidad Católica de Cuenca, Facultad de Ingeniería y Arquitectura, Cuenca Ecuador, mavila@ucacue.edu.ec, https://orcid.org/0000-0002-2134-1432

${ }^{3}$ Universidad Católica de Cuenca, Facultad de Economía, Cuenca Ecuador, ycastilloo@ucacue.edu.ec, https://orcid.org/0000-0002-7710-5199
} 


\section{Resumen.}

En el Ecuador desde el año 2010 se ha venido dando experiencias planificadoras a través de los planes de desarrollo y ordenamiento territorial a cargo de los Gobiernos Autónomos Descentralizados en el caso del cantón Gualaceo ubicado en la parte oriental de la provincia del Azuay. Objetivo. fortalecer e implementar estrategias para un ordenamiento territorial que garantice su sostenibilidad. Metodologìa. Una vez revisado el marco teórico de la planificación y ordenamiento territorial es necesario reflexionar su cumplimiento con la evaluación del PDOT en un período determinado 2015-2019, en base a una revisión documental, encuestas y entrevistas de la percepción de sus autoridades y técnicos de planificación. Resultados. Del análisis de la investigación, evidenciando un bajo grado de cumplimiento en cada una de las estrategias planteadas en la fase de la propuesta referente al modelo territorial y el modelo de gestión, lo que permite establecer una propuesta de la estrategia ambiental y el fortalecimiento de las estrategias planteadas en sus planes. Conclusión. Para garantizar un ordenamiento territorial sostenible comprendiendo en la visión planteada del cantón con liderazgo gubernamental conscientes del conocimiento de los potenciales y debilidades de su territorio.

Palabras claves: planificación, desarrollo local, ordenamiento territorial, estrategias de gestión.

\section{Introducción.}

En el Ecuador el Plan Nacional de Desarrollo se construyó en el año 2007 representando el primer ejercicio de planificación integral a escala nacional, incorporando al ordenamiento territorial como política de Estado con un régimen de competencias en la formulación de la planificación nacional a cargo del estado central y la planificación del desarrollo y planes de ordenamiento territorial, a cargo de todos los niveles de Gobiernos Autónomos Descentralizados y a partir del año 2010 la Secretaría Técnica de Planificación definieron lineamientos con una guía metodológica para la formulación de los planes desde el año 2011, y en el 2015 contando con planes aprobados y actualmente se encuentran realizando las respectivas actualizaciones.

Dentro de la metodología para los Planes se plantea una propuesta de desarrollo como un escenario deseado encontrando la categorías de ordenamiento territorial en función de las variables estratégicas y de potencialidades territoriales del diagnóstico sobre la capacidad de uso de la tierra, su vocación agro-productivas con otras variables de gran importancia de acuerdo a la visión del territorio, planteando un modelo de gestión con la aplicación de estrategias necesarias para su implementación, pero en qué medida han aportado en el ordenamiento territorial sostenible del territorio o se han quedado simplemente en documentos obligatorios sin integración ni la aplicación de las estrategias planteadas. 
Objetivos: Establecer estrategias de ordenamiento territorial sostenible para el cantón Gualaceo, estudiando las teorías sobre la planificación y ordenamiento territorial con criterios de sostenibilidad en el ámbito local, evaluando la planificación y ordenamiento territorial en el cantón Gualaceo 2015-2019, para establecer y fortalecer las estrategias para el ordenamiento territorial sostenible con sus elementos que la componen.

\section{Fundamentación teórica de la investigación.}

Las definiciones emitidas por diferentes autores en las últimas décadas nos permiten conocer y entender los conceptos de la planificación, desarrollo local y ordenamiento territorial a fin de llegar a establecer las estrategias.

Planificación: Para Saavedra 1993, Friedmann 1987, Yehezkel 1990, Habermas 1998, Kozikowski 1998, Galilea 1998 y Kaplan 1989, lo resumen con elementos comunes considerando no solamente como una disciplina sino como un proceso que se realiza en un tiempo y espacio con una visión conocida como imagen objetivo de un futuro deseado.

Anton et ál (2005, p.45) menciona a la planificación con diferencias de la planificación estratégica encaminada a la acción, mientras que la territorial marca un escenario de futuro y dispone de capacidad normativa, así tambien Blades (2011, p. 14) ha presentado tres paradigmas de la planificación tales como planificación del desarrollo que tiene en su origen una fundamentación y una lógica diferente como: planificación estratégica que alcanza el objetivo deseado con ventajas competitivas, la planificación participativa con el compromiso y validación social y la planificación integral del desarrollo basado en la visión del territorio, relación con el entorno y su ubicación dentro del un contexto global en el largo y mediano plazo, es así para Galilea ( p.67) ha considerado la escala local en la planificación y en las acciones del desarrollo lo que hace que las propuestas de planificación local tengan un mayor horizonte de factibilidad sociopolítico, económico y estricatametne técnico.

Desarrollo Local: Vázquez-Barquero (1988; p129) lo define como un proceso de crecimiento económico y de cambio estructural que conduce a una mejora en el nivel de vida de la población local, identificando tres dimensiones: económica, sociocultural y político-administrativa. Algunos autores al sustantivo desarrollo le acompaña algunos adjetivos para Boisier (1999 p.8-21) desarrollo territorial, regional, local, endógeno, descentralizado de abajo-arriba, para Rodriguez (2009 p. 40) contempla además un desarrollo sostenible, Juárez $(2013$ p.19, 20) lo menciona directamente como desarrollo local relacionado con el desarrollo endógeno y sostenible, para Albuquerque (2014, p.6) aborda al desarrollo con dimensiones del desarrollo social y humano, desarrollo institucional, político y cultural, desarrollo económico local y desarrollo sostenible, abordando el enfoque del desarrollo territorial que identifica y aprovecha los recursos locales endógenos así como incorpora políticas tomando en cuenta los diferentes ámbitos global y local. 
Actualmente el concepto de desarrollo local es utilizado en numerosos documentos oficiales teniendo un problema entre su función teórica y la realidad práctica, revalorizando el espacio local como agente productivo global con mejoras en la comunicación social y en el desarrollo sostenible valorando los recursos endógenos con la participación de la población siendo una herramienta que busca mejoras en sus potencialidades.

Ordenamiento Territorial: La ordenación de un territorio ha tomado fuerza últimamente ante los nuevos desafíos territoriales y los cambios en el contexto político social de la planificación ajustada a una realidad natural que es concebida por diferentes autores como Anton et ál (2004, p.16) y Gómez (1994) con un enforque metodológico menciona que ordenar el territorio implica dar respuesta a interrogantes tales como: qué, para qué y cómo ordenar, siendo en sí misma más que una finalidad un instrumento al servicio de los objetivos generales de equidad territorial y el uso racional de los recursos. Así también Terán (1983), citado por Troitiño (2008), y (Sánchez, 2014, p.11) mencionan que la ordenación del territorio alcanzará el desarrollo equilibrado de las regiones y la organización física del espacio según un principio rector.

Estos conceptos van configurando el sistema territorial con la construcción planificada orientada a conseguir un desarrollo sostenible identificando, distribuyendo y regulando de manera armónica y funcional todas las actividades humanas dentro de un territorio, coincidiendo con Malo (2012), Gómez (2014) y Gudiño (2015) que la nueva concepción del ordenamiento territorial se considera no solo en el estudio interdisciplinario del territorio y la planificación, siendo el Estado la entidad político-territorial que permita gestionar el territorio de una manera más eficiente identificando los conceptos para la aplicación de la sostenibilidad en la acción y el cometido de las políticas de planificación territorial, con la utilización racional del territorio, la gestión responsable de los procesos ecológicos, conservando la calidad ambiental y la conservación de ecosistemas y la calidad de gestión pública y la coordinación administrativa con una planificación integrada de sectores y territorios.

La implementación de un ordenamiento territorial sostenible según Folch (2003) pone en énfasis en los principios de impacto sobre el medio ambiente, más que las actividades en el espacio; sobre los ritmos y los procesos de transformación, más que sobre el tipo de transformación; sobre el control de las redes de interrelación, además del control de los elementos individuales; sobre diferentes escalas geográficas, ámbitos sectoriales y horizontes temporales; y sobre la participación de los agentes socioeconómicos y políticos que intervienen en los procesos de transformación del territorio.

En la década del 2000 comienza a tener fuerza el tipo de ordenamiento activo inducido por políticas de ordenamiento que buscan un desarrollo económico con enfoque territorial en el marco del desarrollo sostenible, es así que adquirió fuerza jurídica Latinoamérica es así en Honduras (2003), Uruguay (2008), Ecuador (2010) y El Salvador (2011) al aprobarse leyes en esta dirección. A partir del 2010 la tendencia al Desarrollo Territorial 
sostenible se consolida y amplía a otros países como Argentina, Nicaragua y Guatemala, en los que está en proceso la aprobación de leyes nacionales que van en la misma dirección.

Los cambios más notorios son en los últimos diez años según Cabezas (2008) cuando se observa un acercamiento entre las políticas de desarrollo económico, de ordenamiento territorial y ambiental, a partir de la adopción del concepto de desarrollo sostenible, en países donde han ocurrido cambios políticos y de concepción del desarrollo, especialmente contra el orden económico, social, cultural y territorial siendo los casos de Ecuador, Bolivia, Uruguay, Argentina, Venezuela, Nicaragua y el El Salvador en respuesta a los problemas de iniquidad socio territorial, destrucción de las condiciones ambientales, destrucción de los valores éticos y políticos, adoptando nuevas visiones de desarrollo rompiendo los preceptos de neoliberalismo danto modelos alternativos de desarrollo en los que el ordenamiento territorial se revienta como desarrollo territorial sostenible, surgiendo como concepto como política pública y como estrategia del desarrollo.

Estrategias de planificaciòn para el ordenamiento territorial sostenible a nivel local: Contreras (2013) Smith (1977) define a la estrategia como la fórmula para obtener éxito en el mundo de los negocios, un plan para conseguir los mejores resultados de los recursos y para Schendel y Hofer (1978), citados por Contreras (2013) la estrategia corresponde al modelo fundamental de despliegue de los recursos presentes y futuros; por tanto la estrategia trata de asegurar la supervivencia y prosperidad de un objeto a través de su implementación, para cumplir las expectativas de las partes interesadas en un futuro incierto (Grant, 2005, citado por Contreras, 2013). El conjunto de acciones combinadas que emprenden dirección y que pretenden lograr un fin, o luchar por una misión o visión a corto, mediano y/o largo plazo (Thompson y Strickland, 1999, citados por Contreras, 2013).

Todos estos conceptos de estrategia han ido transformándose de forma progresiva desde objetivos, propósitos, metas, planes, guías, entre otros, y que de una forma sirven como referentes históricos, considerando algunos aspectos y teniendo claro hacia dónde se quiere ir, dónde se quiere llegar y con qué recursos se cuenta para la obtención de resultados, con el uso adecuado de los recursos en el menor tiempo posible.

Según Silva (2012) presenta tipos de estrategias a nivel local visualizando enfoques como estrategias de complementariedad, consolidación, diversificación, recuperación, revalorización, reequilibrio, atracción, integración y de la comunidad, siendo todos estos tipos de estrategias que contribuyen al desarrollo sostenible del territorio, Contreras (2013) concluye que una estrategia implica conocer la actividad con un análisis prospectivo para el logro de los objetivos, teniendo claro hacia dónde se quiere ir, dónde se quiere llegar y con qué recursos se cuenta para obtención de resultados conociendo los riesgos internos y externos para las tomas de decisiones, al trazar las estrategias considerando la capacidad y el cambio en el menor tiempo posible. 
Rodríguez (2011) define a la estrategia en el ámbito técnico define como un conjunto de recursos utilizados por un equipo profesional multidisciplinario para la ejecución de tareas en un determinado espacio social y socio-cultural para producir cambios en los ámbitos planteados, para el diseño de estrategias de intervención se identifica el contexto del problema, su identificación y análisis con causas, efectos para plantear los objetivos generales y específicos, seleccionando las técnicas y recursos para la intervención con la identificación clara de metas y logros.

A nivel latinoamericano los países se van consolidando teniendo una visión global según 2019 "Nuestra Propia Agenda sobre Desarrollo y Medio Ambiente", en el cual se hace un diagnóstico de la realidad latinoamericana y se proponen las bases para la construcción de la estrategia de desarrollo sustentable de los países latinoamericanos. Dichas bases la constituyen 7 grandes líneas maestras: 1) La erradicación de la pobreza. 2) El aprovechamiento sostenible de los recursos naturales. 3) el ordenamiento del territorio. 4) El desarrollo tecnológico compatible con la realidad social y natural. 5) Una nueva estrategia económica y social. 6) La organización y movilización social y 7) La reforma del Estado, es decir al ordenamiento territorial se plantea como una estrategia para lograr el desarrollo sostenible, basada en la "distribución geográfica de la población y sus actividades de acuerdo con la integridad y potencialidad de los recursos naturales que conforman el entorno físico-biótico.

Para López (1995) una estrategia no es un plan, ni siquiera un plan de largo plazo, aunque la planificación sea parte importante de una estrategia, ya que un plan es lineal, rígido y frecuentemente sectorial, una estrategia debe ser dinámica ya que se trata con gran diversidad de grupos y de interés sujetos a una situación dinámica. Las estrategias son adaptativas y gradualmente van construyendo con un objetivo en determinado plazo y es participativa con acuerdos intersectoriales, aprovecha las oportunidades y fortalece las capacidades ya existente, encuentran prioridades, reanalizan periódicamente su situación y mantiene un sistema de seguimiento interno sus acciones van gradualmente cambiando la realidad sin perder el enfoque de proceso, con la aplicación de estrategias tenemos se consigue mejores resultados concluyendo que no existe receta para su aplicación sino la adaptación a condiciones locales, cuanto menor es la jerarquía local mayor posibilidad de aplicación con participación más amplia y resultados más tangibles, siendo necesario actuar en paralelo a niveles locales y nacionales.

Para Aguilar et al. (2011), Salinas (2013, p.144) es como un instrumento que planea y programa el uso del territorio, actividades productivas, ordenacion de asentamientos humanos y el desarrollo de la sociedad de conformidad con el potencial natual aprovechando de manera sustentable los recursos naturales y humanos con la protección y calidad del medio ambiente.

Según, Secretaría Técnica PLANIFICA ECUADOR (2019) dentro del Modelo de Gestión contiene cuatro ejes de estrategias tales como la de articulación y coordinación 
de programas y proyectos según competencias, las estrategias para la reducción progresiva de los factores de riesgo o su mitigación, la estrategia del seguimiento y evaluación, la estrategia de promoción y difusión.

\section{Metodologia.}

La presente investigación contempla las bases con la formulación de teorías sobre la planificación, desarrollo local, ordenamiento territorial y estrategias, seguido de un diagnóstico situacional del caso de estudio con la revisión documental y recolección de información en un período determinado, con una investigación mixta con enfoque cuantitativo y cualitativo.

En la evaluación de la información documental se procede al análisis de la información mediante matrices de cumplimiento de la propuesta existente, así también se ha considerado la percepción de autoridades y técnicos de los Gobiernos Autónomos Descentralizados del cantón, aplicando encuestas y entrevistas dentro del período evaluado 2015-2019 que conlleva una administración municipal anterior y la vigente desde mediados del 2019, considerando que la población es pequeña de autoridades y técnicos dentro del período administrativo que comprende diez concejales del Gobierno Cantonal y dieciséis presidentes de las ocho parroquias rurales se toma un valor de representatividad del $60 \%$ con quince autoridades para la aplicación del instrumento de encuestas cerradas aplicadas, así también entrevistas semiestructuradas dirigidas al 100\% en una muestra de seis técnicos que comprende los responsables de la Planificación dentro de la Municipalidad.

Con el procesamiento de toda la información obtenida ser procede al análisis de la información con alcance descriptivo analítico mediante cruce de los resultados para la formulación de la propuesta como respuesta a la solución de los resultados obtenidos, a fin de mejorar las estrategias existentes y formulando nuevas estrategias para garantizar un ordenamiento sostenible del cantón.

\section{Resultados.}

Caso de Estudio: El cantón Gualaceo está situada en la parte oriental de la provincia del Azuay a una distancia de $35 \mathrm{Km}$ de Cuenca la capital provincial, conformado por nueve parroquias: ocho rurales Mariano Moreno, Daniel Córdova Toral, Luis Cordero Vega, Remigio Crespo Toral, Jadán, Zhidmad, San Juan y Simón Bolívar y una urbana Gualaceo, cantonizada el 25 de junio de 1824 con 196 años de fundación, con una superficie de $345.48 \mathrm{Km} 2$. en las partes más bajas la altura territorial alcanza a $2100 \mathrm{msnm}$ y en sus páramos de altitud 4000 msnm. Población proyectada a 2020 de 49105 total de habitantes, según CPV 2010, Densidad Poblacional de 115,36 h/km2. con principales actividades agricultura, la artesanía y turismo con grandes atractivos naturales y naturales como los ríos Sta. Bárbara y San Francisco, así también zonas protegidas como el bosque Aguarongo y el Collay, declarado como Patrimonio Cultural de la Nación en diciembre de 2001. 
Evaluación de la planificación y ordenamiento territorial en el cantón Gualaceo 2015-2019: En los años 2001-2013 se contaba con el Plan de Desarrollo Estratégico del cantón conjuntamente con el Plan de Ordenamiento Territorial únicamente de la cabecera cantonal que se encuentra vigente hasta la presente fecha. En el año 2013-2015 se formula el PDOT sin tener documentación de soporte, desde el año 2015-2019 se cuenta con el PDOT Cantonal por lo que se realiza la Evaluación de este período en el ámbito de la propuesta y modelo de gestión.

El Modelo Territorial: basado en la zonificación territorial por las categorías de ordenación, red de asentamientos humanos jerarquizados y de comunicación, así como los elementos que condicionan las actividades humanas como (ABVP Bosques Protectores y Reservas Municipales)._Mediante las categorías de ordenación identificadas y clasificadas de acuerdo a cuatro niveles de actuación sobre el medio físico según Cuadro 1 y Gráfico 1; con el Nivel de Uso: Producción 40.42\%, Conservación42.42\%, Recuperación 13.89\%, Expansión 0.97\% y Áreas Urbanas 0.04\%, que describe 14 Categorías de Ordenamiento Territorial contemplada en la ordenanza temporal del área rural con un lote mínimo general; pero dicha zonificación no ha sido contrarrestada con el verdadero deslinde predial ni características propias de los centros poblados que apenas desde el año 2018 está siendo registrado digitalmente, las zonas de expansión contempla únicamente al suelo rural de la parroquia Gualaceo y no así de las demás parroquias rurales, existiendo un crecimiento desordenado en los corredores de crecimiento a lo largo de vías inter parroquial, así como áreas junto al límite urbano parroquial al no existir actualizado los planes de ordenamiento urbano de las parroquias y del mismo centro cantonal.

\begin{tabular}{|c|c|c|c|}
\hline NIVEL DE USO & CATEGORIAS DE ORDENACIÓN & ÁREA (Ha) & $\% \mathrm{Ha}$ \\
\hline \multirow{3}{*}{ Producción 40,42\% } & Zonas Agrícolas & $3.221,27$ & 9,04 \\
\hline & Zonas Agropecuarias & $8.282,38$ & 23,25 \\
\hline & Zonas Silvopastoriles & $2.897,37$ & 8,13 \\
\hline \multirow{5}{*}{ Cons ervación 42,43\% } & Zona de Conservación-Comuna Uchucay & 17,28 & 0,05 \\
\hline & Zonas de Conservación Natural- Páramo & $3.102,60$ & 8,71 \\
\hline & Zonas de Conservación Natural-Vegetación & $8.353,74$ & 23,45 \\
\hline & Zonas de conservación de cauces de ríos y qubradas & $2.157,68$ & 6,06 \\
\hline & Zonas de conservación de Recarga Hídrica & $1.481,09$ & 4,16 \\
\hline \multirow{3}{*}{ Recuperación 13,89\% } & Zonas de Recuperación Ambiental & $3.730,15$ & 10,47 \\
\hline & Zonas de Recuperación Ambiental Bosque Aguarongo & $1.211,92$ & 3,40 \\
\hline & Zona de Riesgos & 5,86 & 0,02 \\
\hline \multirow{3}{*}{ Expansión 0,97\% } & Suelo Rural de Expansión & 15,18 & 0,04 \\
\hline & Suelo Rural de Expansión Urbana & 284,24 & 0,80 \\
\hline & Zonas Industriales & 47,34 & 0,13 \\
\hline Urbana $2,30 \%$ & Cabecera Urbana Cantonal y Parroquial & 819,78 & 2,30 \\
\hline \multicolumn{2}{|r|}{ total } & $35.627,88$ & 100,00 \\
\hline
\end{tabular}

Cuadro 1. Niveles de Uso y Categorías de Ordenación

Fuente: Elaboración Propia en base a PDOT 2015 
NIVELES DE USO

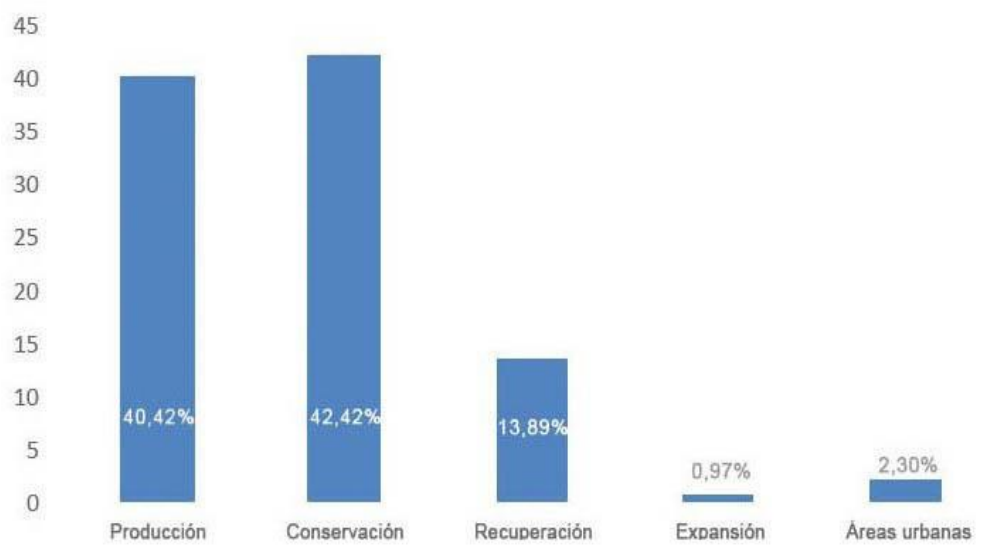

Gráfico 1: Niveles de Uso

Fuente: Elaboración Propia en base a PDOT 2015

La red de asentamientos se encuentra debidamente jerarquizados, la red de infraestructura vial será necesario sea actualizada contando con una cartografía que no solo contemplen las vías principales sino además los caminos públicos y/o vecinales de las localidades con un alcance a las demás Instituciones tales como Subsecretaría de Tierras en la legalización de terrenos en el área rural, en la propuesta está planificado una vía de cuatro carriles de comunicación con la ciudad vecina Cuenca, la misma que no se ha realizado ni estudios ni ha sido contemplada en programas y proyectos, la Circunvalación Bullcay-Gualaceo cuenta con estudios desactualizados por varios años sin que tampoco conste en el listado de proyectos.

Las categorías de ordenación territorial, frente al modelo territorial propuesto no logró clasificar plenamente el territorio del cantón, sin definición de los suelos de expansión urbana y rural dejando vacíos en estas categorías que serían abordadas en la actualización del plan de uso y gestión del suelo.

El Modelo de Gestión establecidos en el PDOT con estrategias planteadas de:

$\checkmark$ Estrategias de coordinación y articulación para la planificación y gestión de los planes.

Política Pública Local. - Programas y Proyectos: con 23 programas y 41 proyectos planteados (políticas, objetivos, metas e indicadores) y su cumplimiento según Gráfico 2, se evidencia los programas realmente ejecutados en los cuatro años con un nivel de cumplimiento general del $50 \%$ y con mayor porcentaje de incumplimiento en los componentes Asentamientos Humanos del 70\% y Político Institucional del 60\%. 
CUMPLIMIENTO DE PROGRAMAS Y PROYECTOS

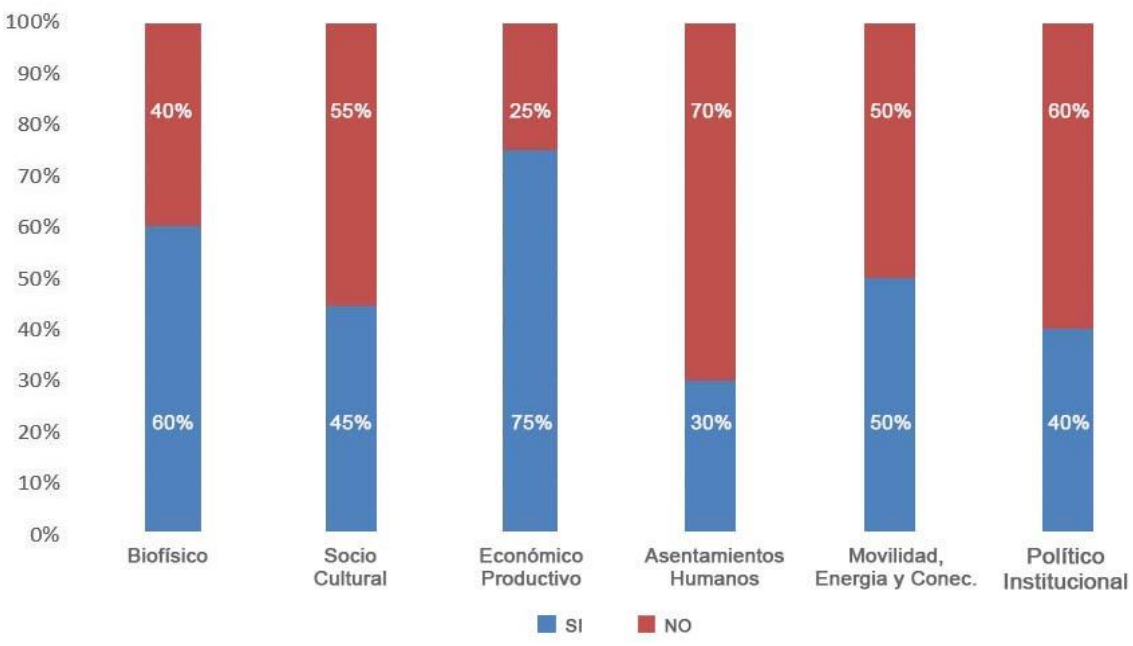

Gráfico 2. Cumplimiento de Programas y Proyectos Fuente: Elaboración Propia en base a PDOT 2015

Política Pública Local. - Agenda Regulatoria: Las estrategias con orientaciones normativas a través de 25 ordenanzas, acuerdos y resoluciones con especificación de objeto y alcance según Gráfico 3., tiene un nivel de cumplimiento general del $44 \%$ y por ende realizadas el $66 \%$, se evidencia el $100 \%$ de cumplimiento en los componentes sociocultural y económico productivo mientras que los restantes alcanzan un $50 \%$ de cumplimiento.

\section{CUMPLIMIENTO DE AGENDA REGULATORIA}

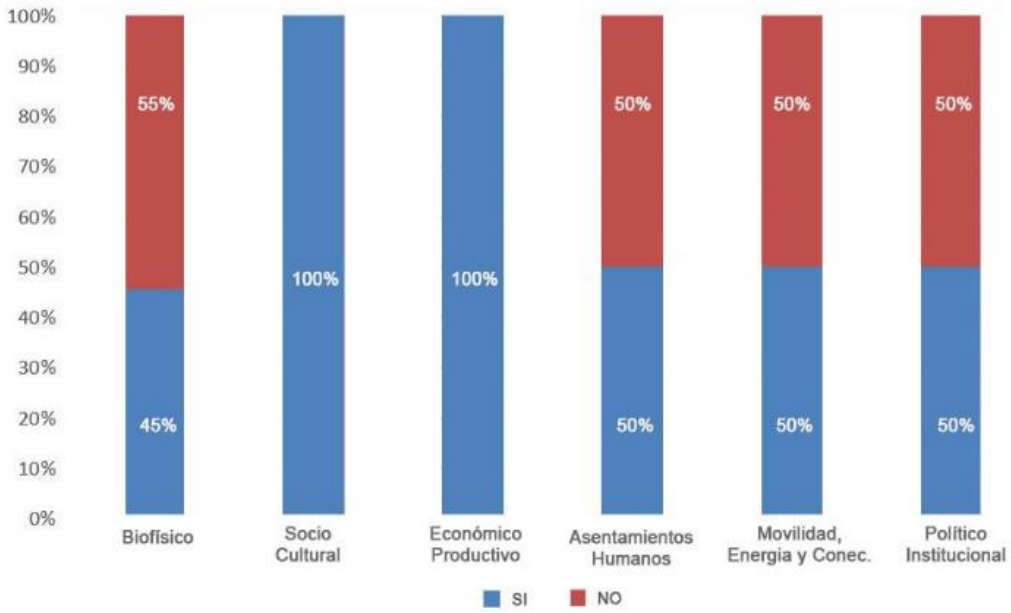

Gráfico 3. Cumplimiento de Agenda Regulatoria Fuente: Elaboración Propia en base a PDOT 2015

Estrategias de Articulación. - Programas y Proyectos: Las 31 estrategias de coordinación y articulación determinadas según Gráfico 4. alcanzan un cumplimiento general de apenas el 24\%, siendo los componentes Biofísico y Socio Cultural con el 75\% y $68 \%$ respectivamente y un incumplimiento total en los demás componentes, pese a existir estrategias planteadas en cada una de los componentes no han sido aplicadas ya que los indicadores de gestión son muy generales así como el tiempo de ejecución, no existe 
estrategias planteadas para los programas y proyectos en el programa de Planificación Territorial como Planes de Ordenamiento Urbano de la Cabecera Cantonal y Cabeceras Parroquiales que no han sido actualizados desde el año 2000 y la Actualización de los Planes de Desarrollo y Ordenamiento Territorial del cantón se han realizado de conformidad con la ley y actualmente se encuentran en proceso; así también si se ha planteado en la Agenda Regulatoria la Ordenanza de Uso y Ocupación del suelo rural pero no se ha ejecutado contando aún con una Ordenanza Temporal muy general.

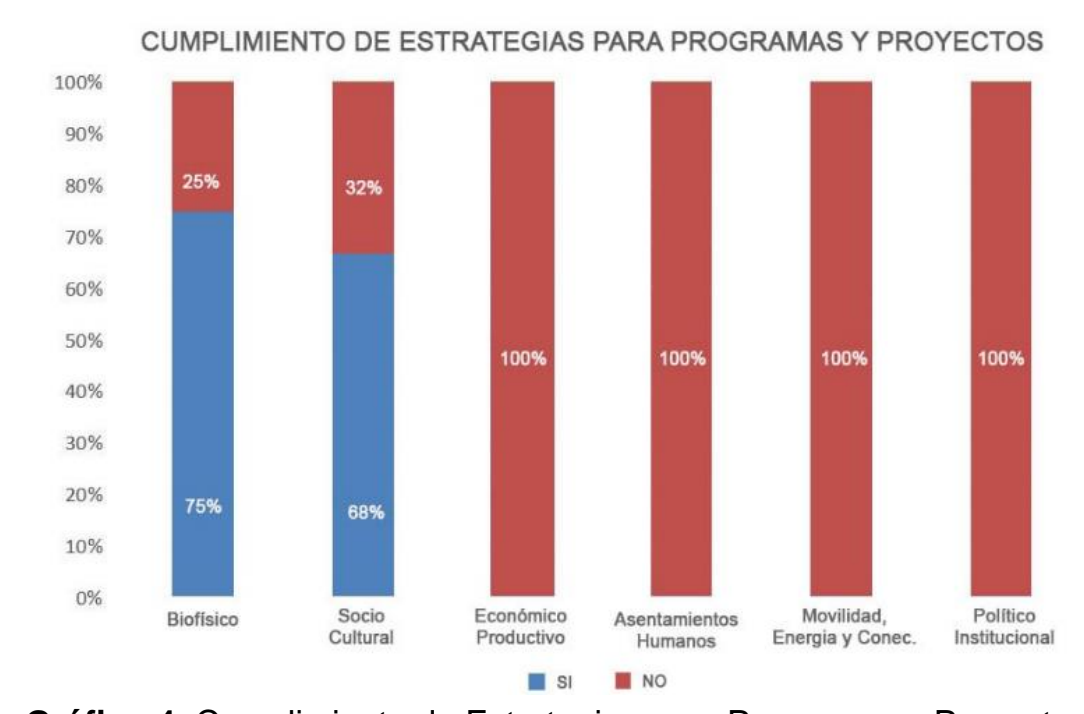

Gráfico 4. Cumplimiento de Estrategias para Programas y Proyectos

Fuente: Elaboración Propia en base a PDOT 2015

Las estrategias de coordinación, articulación y gestión planteado en proyectos, ordenanzas y estrategias alcanzan un nivel bajo del $47 \%$ ya que no existe evaluación ni seguimiento de lo planteado ni han definido los responsables para su aplicación, así también no se han construido indicadores de gestión que aporten información de valor para la medición real que no lograron enfocarse para conseguirlos.

$\checkmark$ Estrategias y metodologías de participación de la ciudadanía en el proceso de planificación y ordenamiento territorial.

La participación ciudadana es corresponsable de la obtención de resultados del Plan en el proceso de formulación, ejecución, seguimiento y monitoreo y la evaluación ciudadana, aplicados en los diferentes espacios y mecanismos participativos determinados tales como:

Unidades de Participación: En el proceso de Presupuestos, Rendición de Cuentas y Sistema de Participación Ciudadana, no existe ni se ha formulado la normativa para su aplicación sino es ejecutado de manera general por el equipo técnico coordinador.

Sistemas de Gestión Social Territorial: Plantean un sistema de información local georeferenciada que permita acceso de la información territorial generada por la municipalidad y on-line a todos los ciudadanos; pero esto no ha sido implementado y se encuentran en proceso constructivo desde el año 2018 con el registro de predios rurales realizado externamente. 
Presupuesto Participativo: Se considera la asignación de recursos de acuerdo al plan per no existe Ordenanza del Presupuesto Participativo planteado en la agenda regulatoria del Plan, ni sigue un procedimiento referente comisión, equipo coordinador, técnico y apoyo local, concertación de reglas de juego, sensibilización y socialización, evaluación y retroalimentación, los demás aspectos como organización y desarrollo de la asamblea, aspectos técnicos básicos, criterios de proyectos a priorizar, recopilación de los proyectos presentados por la ciudadanía, presentación de los proyectos al Concejo Cantonal.

La estrategia de participación ciudadana en general, no se ha implementado en su totalidad siendo mínima su aplicación Unidades de Participación y Presupuesto Participativo y nula en los sistemas de gestión social territorial.

$\checkmark$ Estrategias y metodología de seguimiento y evaluación del Plan y de la inversión pública.

El proceso de Seguimiento proporciona alertas tempranas que permitan retroalimentar las políticas públicas en los territorios; pero han sido únicamente ejecutadas a nivel de reportes al Sistema de Información de los GAD a la Secretaría Técnica Planifica Ecuador en lo que corresponde al cumplimiento de metas del PDOT cuyos informes anuales según Cuadro 2 tiene un cumplimiento de metas ICM del 0.77 al 1.00, en avances físicos del 77 al $78 \%$ y un avance presupuestario del 83.60 al $100 \%$ pero se contradice con los resultados obtenidos con la realidad interna al no existir registro alguno de seguimiento y evaluación.

\begin{tabular}{|c|c|c|c|c|c|}
\hline \multicolumn{6}{|c|}{ REPORTES SIGAD } \\
\hline DENOMINACIÒN & 2015 & 2016 & 2017 & 2018 & 2019 \\
\hline Proyectos ingresados $\mathrm{N}$. & 59 & 46 & 53 & 28 & 29 \\
\hline Inversiòn ejercicio fiscal \$ & 6443813,81 & 5307950,33 & 4297923,55 & 7687405,10 & 10595447,70 \\
\hline Indice de cumplim. de metas & 0,77 & 1,00 & $\mathrm{~S} / \mathrm{D}$ & 0,94 & 0,93 \\
\hline Avance Fisico \% & $\mathrm{S} / \mathrm{D}$ & S/D & $\mathrm{S} / \mathrm{D}$ & 77,87 & 77,34 \\
\hline Avance Presupuestario \% & 83,60 & 101,17 & $\mathrm{~S} / \mathrm{D}$ & 100,00 & 93,69 \\
\hline
\end{tabular}

Cuadro 2. Reportes SIGAD

Fuente: Elaboración Propia en base a Registros SIGAD

La estrategia de seguimiento y evaluación del Plan a nivel interno de la Institución no se ha realizado para la implementación e intervenciones físicas de programas y proyectos ni conclusiones ni recomendaciones oportunas y se limita a un registro obligatorio en el Sistema de Información de Gobiernos Autónomos Descentralizados (SIGAD) que evidencia un mayor cumplimiento al registrado internamente.

Según la percepción de autoridades de los Gobiernos Autónomos Descentralizados del cantón y técnicos de planificación, si bien es cierto le atribuyen la importancia con un 87\% a la Planificación y Desarrollo Local del cantón, considerando el 67\% que es aceptable la aplicación de los planteamientos del PDOT, dando una valoración negativa a la estructura organizativa de planificación de su cantón de regular el $66.7 \%$ y pésima el 
$13.3 \%$ y consideran que no se ha llegado aplicar un ordenamiento sostenible mencionando el $40 \%$ que poco se ha aplicado y el $20 \%$ nada, pero a la vez en su mayoría se evidencia un desconocimiento general del PDOT en lo que respecta a la visión cantonal con el $73.3 \%$, el ordenamiento territorial con el $53.3 \%$ poco y el $26.7 \%$ nada conocen, así también las estrategias el $46.7 \%$ poco y el $40 \%$ nada conocen al respecto, la agenda regulatoria el $33.3 \%$ poco y el 33.3 así también mencionando el $80 \%$ que no ha existido un seguimiento ni evaluación de la propuesta planteada; pero manifiestan en su mayoría el 80\% es necesario la aplicación de más estrategias es decir atribuyen el éxito y/ o fracaso de la Planificación de su cantón el $46.7 \%$ únicamente al desconocimiento del PDOT interno y externo, y el $46.7 \%$ al mismo desconocimiento del PDOT a nivel interno y externo, así como la falta de estrategias, apoyo de autoridades de turno y personal técnico de planificación.

De igual manera, las encuestas de carácter abiertas para los Técnicos de planificación del Gobierno municipal mantuvieron respuestas similares, resaltando que, el PDOT no es tomado en cuenta, debido a que, solo se lo elabora para cumplir con la ley, pero, carece totalmente del interés de las autoridades para su cumplimiento, por lo que, no es conocido a nivel interno ni externo, por eso los informes o reportes no se han llevado a cabo sin el seguimiento ni evaluación respectiva, salvo para cumplir con el SIGAD. Algunas de las respuestas que surgieron de las entrevistas semiestructuradas.

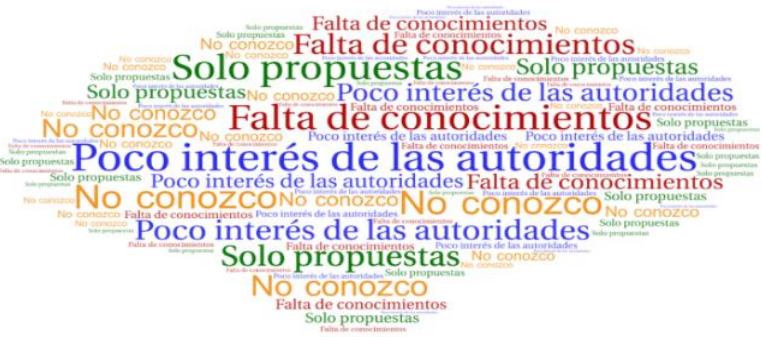

Gráfico 5. Percepción de PDOT Técnicos de Planificación Fuente: Elaboración Propia

El mayor problema que existe en relación al PDOT y por ende de las estrategias planteadas, es la falta de conocimiento de las autoridades, técnicos municipales y la misma población, es notoria la importancia que todos le dan al Desarrollo y Ordenamiento Territorial pero al existir un desconocimiento para su aplicación así como la falta de personal técnico capacitado y poco interés de todos, es necesario fomentar el interés y conocimiento a nivel interno municipal y se pueda proyectar a toda la población del cantón.

$\checkmark$ Estrategia de Difusión del Plan.

La estrategia de difusión se realizó únicamente en el año 2015 cuando se realizaba los planes y en los años siguientes la ciudadanía en general desconoce al igual que la mayoría de sus autoridades y jefes departamentales, ya que carecen de un Plan de Difusión interno y externo debidamente estructurado. 
Propuesta de estrategias para el ordenamiento territorial sostenible: A fin de determinar un ordenamiento territorial sostenible en el cantón, de conformidad con la evaluación del PDOT 2015-2019 referente a las estrategias planteadas, la propuesta Gráfico 6. en la presente investigación contempla en primera instancia el mejoramiento y fortalecimiento de las estrategias ya planteadas en el modelo de Gestión de acuerdo con la guía metodológica para la formulación/actualización del PDOT año 2019, emitida por la Secretaría Técnica de Planificación.

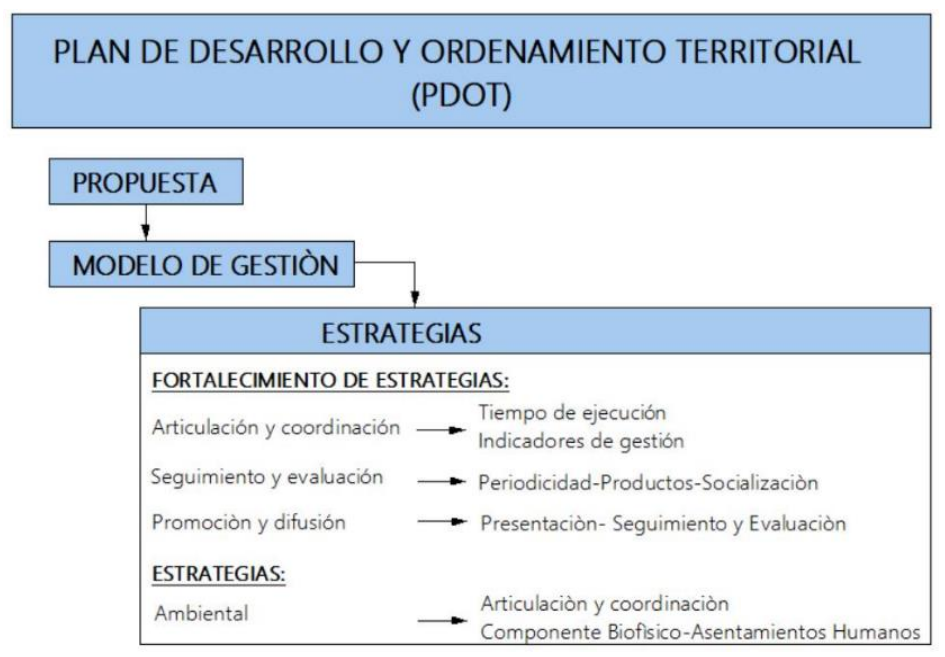

Gráfico 6. Estrategias

Fuente: Elaboración Propia

Cabe señalar que la estrategia de participación ciudadana no se encuentra ahora de manera específica en el modelo de gestión sino se trata de todo un sistema presente en todo el proceso de formulación/actualización e implementación de los PDOT y presente en cada una de las estrategias generales; siendo necesario se desarrolle una metodología de participación ciudadana que involucre a los actores locales: administración municipal, las empresas y/o instituciones con la inversión de recursos y esfuerzos, el involucramiento de la academia con iniciativas de investigación y capacitación y sociedad civil con las organizaciones sociales para que lleven a cabo las acciones necesarias.

\section{$\checkmark$ Fortalecimiento de estrategias de articulación y coordinación para la implementación del PDOT.}

Para la propuesta de las estrategias se aplica los diferentes elementos planteados según Jordá., (2016) que tiene la vocación de transformar el territorio a medio y largo plazo aplicados según el Cuadro 3.

1. una visión, elemento fundamental sin el que no puede existir estrategia.

2. unas líneas estratégicas, aquéllas en las que el territorio va a volcar sus esfuerzos y sus recursos para convertirse en un referente entre los demás territorios.

3. unas actuaciones básicas, o proyectos clave para cada línea, que reforzarán al territorio hasta hacerle alcanzar la excelencia en las líneas estratégicas escogidas.

4. un calendario, que fija los grandes hitos del despliegue de la estrategia, la marcación de plazos de ejecución para los programas y proyectos a corto, mediano y largo plazo estableciendo compromisos con un calendario definido y conocido por los involucrados. 
5. organización, constituida por los actores del territorio y que se encargará de impulsar, coordinar y hacer el seguimiento del progreso de la estrategia con la misión de supervisar y coordinar la puesta en marcha con una estructura profesional con los actores territoriales implicados en la estrategia.

\begin{tabular}{|c|l|l|l|l|}
\hline 1. VISION & 2. LINEAS ESTRATEGICAS & 3. ACTUACIONES BASICAS & 4.CALENDARIO & 5. ORGANIZACIÓN \\
\hline $\begin{array}{l}\text { enmarcada en la } \\
\text { visiòn cantonal }\end{array}$ & $\begin{array}{l}\text { componentes: biofisico, } \\
\text { sociocultural, econòmico- } \\
\text { productivo, asentamientos } \\
\text { humanos y polìtico-institucional }\end{array}$ & estrategia planteada & plazos (meses, años, perìodo) & $\begin{array}{l}\text { Involucrados en la } \\
\text { estrategia: instancias y } \\
\text { responsables, lìneas de } \\
\text { acciòn. }\end{array}$ \\
\hline
\end{tabular}

Cuadro 3. Elementos de la Estrategias

Fuente: Elaboración Propia

\section{$\checkmark$ Fortalecimiento de estrategias de articulación y coordinación para la implementación del PDOT.}

En las 30 estrategias de articulación planteadas para los programas y proyectos en el PDOT en cada uno de los componentes según el Cuadro 4, se ajustan y determinan los indicadores de gestión y se fija el año 1,2, 3 o 4 para su ejecución, ya que actualmente se tiene como tiempo de ejecución 4 años de manera muy general y los indicadores de gestión menciona presupuesto devengado/ presupuesto programado que son datos muy generalizados que no garantizan una retroalimentación oportuna.

\begin{tabular}{|l|l|l|l|l|l|l|l|}
\hline \multicolumn{7}{|c|}{ ESTRATEGIAS DE ARTICULACIÓN SEGÙN COMPONENTE } \\
\hline $\begin{array}{l}\text { PROBLEMA } \\
\text { JERARQUIZADO }\end{array}$ & $\begin{array}{l}\text { ESTRATEGIA DE } \\
\text { ARTICULACIÓN }\end{array}$ & PROGRAMA & PROYECTO & PRESUPUESTO & $\begin{array}{l}\text { FUENTE DE } \\
\text { FINANCIAMIENTO }\end{array}$ & $\begin{array}{l}\text { TIEMPO DE } \\
\text { EJECUCIÓN }\end{array}$ & $\begin{array}{l}\text { INDICADOR DE } \\
\text { GESTIÓN }\end{array}$ \\
\hline
\end{tabular}

Cuadro 4. Estrategias de Articulación según Componente Fuente: Elaboración Propia en base a PDOT 2015

\section{$\checkmark$ Fortalecimiento de estrategias de seguimiento y evaluación del PDOT.}

En esta estrategia se reforzará según Cuadro 3, el punto 5 de Organización en las diferentes Sesiones del GAD Cantonal con las comisiones afines a los componentes del punto 2, con previas reuniones de trabajo del Departamento de Planificación y su unidad responsable del PDOT la misma que deberá ser fortalecida para monitorear acciones tanto a nivel interno como externo siendo el apoyo técnico del Consejo de Planificación y programando talleres con Planifica Ecuador de ser necesario muy a parte de los registros SIGAD obligatoriamente establecidos. El equipo responsable a nivel estratégico lo conforman las diferentes comisiones y a nivel ejecutivo se sugiere la conformación de un equipo interno conformado por los directores departamentales que serán los responsables de realizar el seguimiento y evaluación con el técnico responsable del PDOT.

Seguimiento Mensual: El técnico responsable registrará la matriz de seguimiento y evaluación según la guía de PLANFIICA ECUADOR, que será recopilada y sistematizada para informar al presidente de los avances de proyectos, problemas que presenten en la implementación y las acciones a realizar para avanzar en el cumplimiento de las metas propuestas. 
Seguimiento Trimestral: El equipo responsable a través de reuniones trimestrales de la ejecución del PDOT de programas y proyectos, objetivos y metas (Registro SIGAD) entre los técnicos involucrados y los beneficiarios de que permitan tomar acciones correctivas a tiempo con el involucramiento de la población.

Evaluación Semestral: Reuniones con el Consejo de Planificación Cantonal con integración en el territorio para presentar el estado de cada uno de los programas y proyectos.

Evaluación Anual: Una vez reportados trimestralmente los avances de programas y proyectos del PDOT se cuenta con un reporte anual que deberá ser conocido por las instancias y responsables en la Asamblea para la Rendición de Cuentas.

Con Productos a entregar:

Matriz de seguimiento y evaluación de Programas y Proyectos. Fichas e informes de proyectos para presupuesto participativo. Fichas de beneficiarios de proyectos con la memoria técnica descriptiva. Reportes al SIGAD. Actas de memorias de reuniones de trabajo.

Hoja de asistencia. Actas de sesiones. Informes técnicos de Programas y Proyectos. Informes anuales de Seguimiento al PDOT a ser entregada a la Secretaría Técnica de Planificación. Con Socialización Interna y Externa

\section{$\checkmark$ Fortalecimiento de estrategias de promoción y difusión.}

Presentación del PDOT 2019-2023 difundiendo la VISION Cantonal y los programas y Proyectos planificados y las estrategias del modelo de gestión.

Seguimiento y evaluación del PDOT en donde se difunde los avances y el cumplimiento de las objetivos y metas propuestas.

El Plan deberá ser difundido y promocionado apoyado en insumos comunicacionales como un folleto ejecutivo resumen a ser difundido en la WEB y entregado a los diferentes actores, letreros para la difusión de la VISION en lugares estratégicos y espacios públicos en cada una de las parroquias. La Evaluación del Plan deberá ser difundido en boletines on line del avance en la página WEB y redes sociales

\section{$\checkmark$ Estrategia ambiental.}

Si bien es cierto el cumplimiento de la política ambiental nacional está regulado por el Ministerio del Medio Ambiente las acciones que propician su cumplimiento dependen de los PDOT de tal forma que permitan un desarrollo sostenible en el territorio para que sean viables y controlables de conformidad con las condiciones tecnológicas, financieras, organizativas y humanas de la localidad, por lo que en las nuevas guías 2019 contempla 
una nueva estrategia de reducción progresiva de los factores de riesgo y su mitigación para fortalecer las unidades técnicas de gestión de riesgo para identificar, proponer y gestionar riesgos identificados, siendo necesario establecer un Plan a corto mediano y largo plazo tanto en el área urbana y rural contando con los mapas de amenazas y riesgos actualizados que permitan actualizar el ordenamiento del territorio con programas de reforzamiento y rehabilitación pero es necesario que contemple aspectos más generales es decir sea el planteamiento de la Estrategia Ambiental a fin de contar con un ordenamiento territorial sostenible del cantón con una articulación de los componentes Biofísico y de Asentamientos Humanos.

Conforme la estructura organizacional del GAD Municipal de Gualaceo cuenta con la Unidad de Gestión Ambiental con la Oficina de Riesgos, a través de la cual se deberá canalizar la articulación con las demás entidades competentes y de manera especial con la Unidad responsable del PDOT.

\section{Conclusiones.}

- El PDOT Cantonal adoptado desde el año 2015, contempla bajos índices del cumplimiento en cada una de las estrategias planteadas sin retroalimentación para el mejoramiento continuo, en el ámbito del Modelo de Gestión surge la necesidad urgente de elaborar y fortalecer los elementos de las estrategias que ayuden a verificar su cumplimiento y garantizar una planificación y ordenamiento territorial sostenible.

- Para aplicar las estrategias de debe contar de manera urgente con la Estructura Organizativa de la Municipalidad con el fortalecimiento de la Unidad de Coordinación del PDOT Cantonal con el involucramiento de las autoridades, agentes locales y los técnicos de cada uno de los departamentos municipales a fin de contar con un seguimiento y evaluación a nivel interno que les permita tomar los correctivos oportunamente y sea conocido por toda la ciudadanía, así también cuente a corto plazo con un sistema de información geográfica para garantizar un verdadero ordenamiento territorial, y expedientes de su organización territorial no solo la recopilación estadística, cartografía básica y temática del territorio, sino que sea una herramienta digital para la ciudadanía y de seguimiento de gestión para efectos de poder ajustar, complementar o mantener las actuaciones de planes, programas y proyectos previstos.

- Es necesario un liderazgo gubernamental conscientes del conocimiento de los potenciales y debilidades de su territorio dispuestos a conocer, concebir y ejecutar estrategias relacionadas con la gestión ambiental para garantizar un ordenamiento territorial sostenible comprendiendo en primera instancia la visión planteada.

\section{Referencias bibliográficas.}

Aguilar, M. (2011). Ordenamiento territorial y participación social problemas y posibilidades. 
Antón, S. (2005). Planificación Territorial del turismo

Albuquerque, F. (2014). Evolución del desarrollo territorial.

Boisier, S. (1999). Desarrollo local de qué estamos hablando.

Contreras, E. (2013). El concepto de estrategia como fundamento de la Planeación Estratégica.

Folch, R. (2005). El territorio como sistema: conceptos y herramientas Ordenación.

Galilea, S., La planificación local: nuevas orientaciones, metodológicas.

Gómez, D. (2014). X simposio nacional de desarrollo urbano y planificación territorial.

Jordá, A. (2016). Manual de la Estrategia de desarrollo para ciudades intermedias

Juárez G. (2013). Revisión del concepto de desarrollo local desde una perspectiva territorial.

Malo, C. (2012). Revista de la Universidad del Azuay N. 57 Ordenamiento Territorial y descentralización.

Méndez, H. (2008). Desarrollo de herramientas y estrategias para el Ordenamiento Territorial.

Méndez, H. (2008). Desarrollo de herramientas y estrategias para el Ordenamiento Territorial. Molina Rudolf et ál. (2014). Estrategias y políticas nacionales para la cohesión territorial Estudios de caso latinoamericanos.

Rodríguez, M. (2011). Estrategias de intervención: notas metodológicas

Saavedra, R. (2001). Planificación del Desarrollo.

Sánchez, D. (2004). Principios de Planificación Territorial.

Salinas, E. (2013). Reflexiones acerca del papel del ordenamiento territorial en la planificación y gestión ambiental.

Secretaría Técnica PLANIFICA ECUADOR, (2019). Guía para la formulación/actualización del PDOT Cantonal.

Silva et ál. (2014). Estrategias y políticas nacionales para la cohesión territorial Estudios de caso en América Latina.

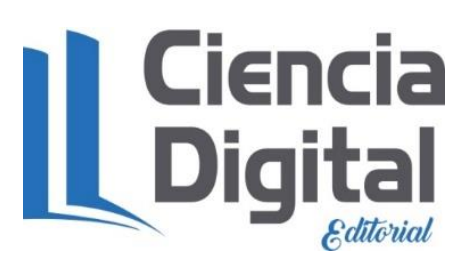




\section{PARA CITAR EL ARTÍCULO INDEXADO.}

Sarango Simbaña, S. M., Ávila Calle, M. B., \& Castillo Ortega, Y. (2021). Estrategias de ordenamiento territorial sostenible para el cantón Gualaceo. ConcienciaDigital, 4(2), 55-73. https://doi.org/10.33262/concienciadigital.v4i2.1627

\section{Liencia}

El artículo que se publica es de exclusiva responsabilidad de los autores y no necesariamente reflejan el pensamiento de la Revista Conciencia Digital.

El artículo queda en propiedad de la revista y, por tanto, su publicación parcial y/o total en otro medio tiene que ser autorizado por el director de la Revista Conciencia Digital.

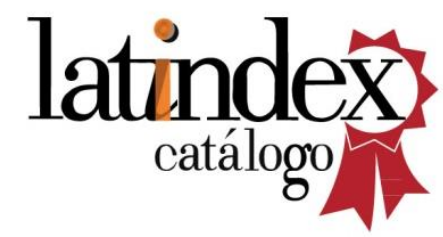

